\title{
Coherencia del modelo pedagógico y la evaluación del aprendizaje en una universidad de Bogotá ${ }^{1}$
}

\author{
Blanca Elpidia Tovar Riveros ${ }^{2}$ \\ Fundación Universitaria del Área Andina, Bogotá, Colombia ${ }^{3}$, \\ betovarr@hotmail.com
}

1 Artículo de investigación resultado de la investigación titulada Modelo Pedagógico y la Evaluación del Estudiante en Enfermería 2013. (Universidad Nacional de Colombia)

2 Enfermera. Fundación Universitaria del Área Andina. Especialista en Cuidado Crítico. Universidad Javeriana. Magister en Educación y Docencia Universitaria, Universidad Nacional de Colombia.

3 Docente universitaria de Enfermería 


\title{
Coherencia del modelo pedagógico y la evaluación del aprendizaje en una universidad de Bogotá
}

\section{Resumen}

Este estudio surge del desempeño profesional como docente universitaria y de la preocupación por la calidad profesional del egresado en Colombia; pues, el profesional en el proceso de enseñanza-aprendizaje debe desarrollar competencias profesionales para un desempeño profesional. Los objetivos fueron: a) indagar sobre la coherencia entre el modelo pedagógico y las prácticas de evaluación al estudiante; b) identificar los elementos del modelo pedagógico vigente; c) conocer el proceso de evaluación del estudiante y d) generar estrategias para mejorar el proceso de enseñanza-aprendizaje. Este estudio se desarrolló en áreas específicas de enfermería. Metodología: estudio descriptivo de cohorte transversal mixto, la recolección de datos: instrumentos como entrevistas semiestructuradas a los docentes y cuestionarios virtuales para los estudiantes. Consecutivamente, se hizo la triangulación entre los resultados de las entrevistas, los cuestionarios y el marco teórico. Los resultados generaron estrategias y conclusiones para mejorar las prácticas evaluativas a partir del modelo pedagógico constructivista.

Palabras claves: Pedagogía, evaluación, competencias, enfermería y ciencias de la salud.

\section{Coherence of the pedagogical model and the evaluation of the learning process in a university in Bogotá}

\begin{abstract}
This research arises from the professional performance as university teacher and the worry by professional quality of the graduate in Colombia, since the graduate must develop professional competences during the teaching-learning process for professional performance. The objectives were: a) to inquire about the coherence between the pedagogical model and the evaluation practices to the student; $b$ ) to identify the elements of the current pedagogical model; c) to know the student's assessment process and d) to create strategies to improve the teaching-learning process. This study was developed in specific fields of nursing. Methodology: descriptive study of mixed transverse cohort, data collection: instruments such as semi-structured interviews to teachers and virtual surveys to students. Consecutively, a triangulation between interviews' results, surveys and theoretical framework was conducted. The results produced strategies and conclusions to enhance the evaluative practices based on the constructivist pedagogical model.
\end{abstract}

Key words: pedagogy, evaluation, competences, nursing and health sciences.

\section{Coerência do modelo pedagógico e a avaliação da aprendizagem numa universidade de Bogotá}

\section{Resumo}

Este estudo surge do desempenho profissional como docente universitária e da preocupação pela qualidade profissional do egresado na Colômbia; pois, o profissional no processo de ensino-aprendizagem deve desenvolver concorrências profissionais para um desempenho profissional. Os objectivos foram: a) indagar sobre a coerência entre o modelo pedagógico e as práticas de avaliação ao estudante; b) identificar os elementos do modelo pedagógico vigente; c) conhecer o processo de avaliação do estudante e d) gerar estratégias para melhorar o processo de ensino-aprendizagem. Este estudo desenvolveu-se em áreas específicas de enfermaria. Metodologia: estudo descritivo de coorte transversal misto, a coleta de dados: instrumentos como entrevistas semi estruturadas aos docentes e questionários virtuais para os estudantes. Consecutivamente, fez-se a triangulação entre os resultados das entrevistas, os questionários e o marco teórico. Os resultados geraram estratégias e conclusões para melhorar as práticas evaluativas a partir do modelo pedagógico constructivista.

Palavras-chave: pedagogia, avaliação, habilidades, enfermaria e ciências da saúde. 


\section{Introducción}

Como profesional del área de las ciencias de la salud, específicamente de enfermería, la investigadora ha tenido la oportunidad de laborar como docente en asignaturas específicas como: Cuidado de enfermería -en el componente teórico como en las prácticas-, en instituciones públicas y privadas. A través de la labor como docente, la autora ha observado que para un estudiante es muy importante el resultado de una evaluación cuantitativa, que lo promueva automáticamente pasar de un semestre a otro $y$, finalmente, obtener un título profesional. No es muy frecuente ver en el proceso evaluativo un enfoque cualitativo que fomente el autorreflexión y el aprendizaje y tal vez los resultados de las evaluaciones no reflejan el proceso de aprendizaje que garantice en un futuro un desempeño profesional competente. Ello llevó a la autora a generar algunos interrogantes de cómo un docente puede ser más participe del proceso de aprendizaje y así lograr que el estudiante tome más conciencia de su aprendizaje para obtener una evaluación más significativa, reflexiva y crítica. En otras palabras, la evaluación debe ser desde el conocimiento, el ser y el hacer de la profesión, para que así sea fácil desempeñarse en diferentes ámbitos laborales. Por tanto, es importante reconocer que la calificación como parte del proceso de evaluación de un estudiante es una herramienta de medición que permite generar conocimiento significativo en la educación superior; que es necesario en cualquier contexto social y no sólo de oportunidad para conseguir una titulación. Sin más preámbulo se describirán las categorías necesarias para conocer si el modelo pedagógico es acorde a la evaluación planteada en el programa de Enfermería.

Un modelo pedagógico es un constructo que agrupa o explica la forma de la educación en una institución, para Flórez es la representación de las relaciones que prevalecen en el proceso de enseñanza-aprendizaje, y la forma de evaluar al estudiante (Flores, 2005). 
Grafica 1. Clasificación de los Modelos pedagógicos.

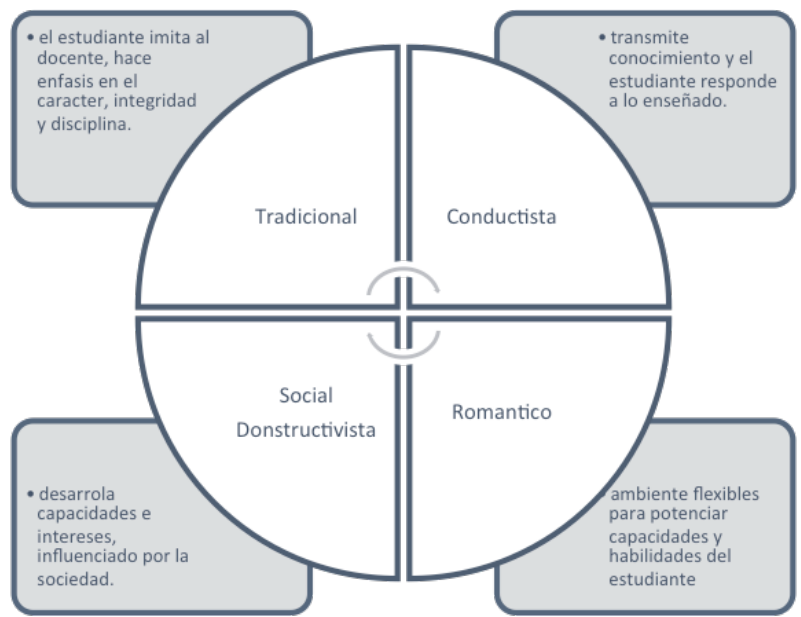

Fuente: (Díaz y Hernández, 2002; Flórez, 2005) Con algunas modificaciones.

Los elementos de un modelo pedagógico son: los contenidos que faciliten un desarrollo cognitivo; la metodología mediante la creación de ambientes que le permitan aprender; el papel del docente que consiste en ser el facilitador o estimulador del aprendizaje (Flores, 2005).

Revisando el modelo pedagógico constructivista, específicamente planteado por el marco de la Universidad, se encontró que para Díaz y Hernández (2002), [el modelo pedagógico constructivista] pretende que el estudiante desarrolle su proceso de aprendizaje de acuerdo a sus capacidades individuales, por ello es importante el ambiente facilitador para que acceda a estructuras cognoscitivas transformadoras y Ilegue a reflexionar sobre su aprendizaje.

Un modelo pedagógico y la evaluación en Enfermería requiere de un proceso de enseñanza y aprendizaje en el que el estudiante realice búsquedas bibliográficas, analice la información y para construir su propio conocimiento debe ser reflexivo, argumentativo y crítico para resolver problemas reales o toma de decisiones; para lo que se debe evaluar por procesos con retroalimentación permanente del avance (Flores, 2005).

La Universidad propone el modelo constructivista desde el currículo y el plan de estudios, además, desarrollar proceso de enseñanza-aprendizaje que permita alcanzar competencias del ser, del saber, procedimentales y comunicativas. Cómo enseñar Orientando el proceso a partir de los intereses de los estudiantes, teniendo en cuenta el conocimiento profesional del profesor. La manera de lograr el aprendizaje 
significativo es mediante la evidencia de la construcción y transformación de estructuras conceptuales, actitudinales, prácticas y axiológicas, y la capacidad de interactuar con el otro. ¿Para qué enseñar? Para lograr un estudiante que pueda desenvolverse en cualquier contexto de su ejercicio profesional.

En cuanto al concepto de la evaluación, siempre que se habla o se reflexiona frente a ella en educación, inmediatamente se piensa y se relaciona con examen o prueba, este proceso también recuerda experiencias desagradables que se han tenido en relación con resultados o calificaciones obtenidos durante el proceso de enseñanza y aprendizaje. El concepto de evaluación va más allá de una calificación, aunque esta se hace para obtener y conocer el proceso formativo del estudiante y así ratificar la calidad del aprendizaje (Doblez, M.C., et al., 1995).

La Ley general de educación (Ley 115 de 1994), apoyada en la Constitución política de Colombia, respecto al derecho a la educación señala:

La evaluación de la educación, el sistema diseñará y aplicará criterios procedimientos para evaluar la calidad de la enseñanza que se imparte, el desempeño profesional del docente, y de los directivos, los logros de los alumnos, la organización administrativa y física de la instituciones educativas y la eficiencia de las prestación del servicio (Art. 80).

La evaluación es la manera de verificar un proceso o momento donde se necesite un criterio para elegir o seleccionar algo. Para hablar de unos componentes del rompecabezas de la educación es relevante y de gran importancia la evaluación, la que se ha convertido en la forma de asegurar la calidad del perfil del futuro profesional. Para dar inicio a esta parte del proceso de evaluación se presentan algunos conceptos de lo que puede ser una evaluación al estudiante: Es la manera de emitir juicios sobre el desempeño de las personas, también para verificar el desarrollo de programas e instituciones, esta evaluación se hace a través de la observación y la investigación (Gette, 2007).

Para Sanmartí (2007), la evaluación es un proceso que permite recoger información, analizarla, tomar decisiones y emitir un juicio. La Joint Committe on standars for Educational Evaluation (1981) la define como una manera de emitir juicios para dar validez a un objeto en cuestión.

Para Moreno (2010), la postura epistemológica del para qué sirve la evaluación y hacia dónde orientar el proceso de enseñanza-aprendizaje; cuyo fin es hacer retroalimentación al estudiante para detectar dificultades y reforzar el aprendizaje. 
Tabla 1. Funciones y enfoques de la evaluación.

\begin{tabular}{|c|c|c|c|}
\hline Diagnóstico & $\begin{array}{l}\text { Permite identificar las } \\
\text { capacidades cognitivas y } \\
\text { especificas generales del } \\
\text { estudiante. }\end{array}$ & $\begin{array}{l}\text { Juicio de } \\
\text { experto }\end{array}$ & $\begin{array}{l}\text { Realizada por un experto } \\
\text { y conocimiento en el área } \\
\text { específica. }\end{array}$ \\
\hline Instructiva & $\begin{array}{l}\text { Genera estrategias e incor- } \\
\text { pora experiencias. }\end{array}$ & $\begin{array}{l}\text { Congruencia } \\
\text { entre objetivo } \\
\text { y desempeño }\end{array}$ & $\begin{array}{l}\text { El evaluador establece criterios } \\
\text { para valorar el desempeño. }\end{array}$ \\
\hline $\begin{array}{l}\text { Educativa } \\
\text { Y pedagógica }\end{array}$ & $\begin{array}{l}\text { Es una estrategia para } \\
\text { erradicar problemas del } \\
\text { desempeño del estudiante. } \\
\text { Permite regular la enseñan- } \\
\text { za y revisar estrategias para } \\
\text { la enseñanza. }\end{array}$ & $\begin{array}{l}\text { Toma de deci- } \\
\text { siones }\end{array}$ & $\begin{array}{l}\text { Permite lograr la decisión más } \\
\text { adecuada, también recolec- } \\
\text { ción y análisis de resultados. }\end{array}$ \\
\hline Autoformadora & $\begin{array}{l}\text { Orienta y genera responsa- } \\
\text { bilidad al estudiante sobre } \\
\text { su propio aprendizaje }\end{array}$ & & \\
\hline
\end{tabular}

Fuente: (Moreno, 2010; Díaz B. y Hernández, 2002; Mora, 2004; Parra, 2008) Con algunas modificaciones.

Lo anterior evidencia que la literatura tiene un constructo claro del para qué y por qué de la evaluación, no sólo con el mero objetivo de sumar y acceder a una titulación. La universidad plantea una evaluación por competencias y revisando se evidenció lo siguiente:

\section{Evaluación por competencias}

Las competencias hacen referencia a todo conjunto de conocimientos, habilidades, destrezas, actitudes y valores que se requieren para desempeñar con éxito o con un determinado nivel de perfección, tarea que responda a las necesidades de una realidad especifica (Cerda, 2000).

Para Pinilla (2007), el término de competencia se refiere a la condición de un profesional y la capacidad de resolver problemas en un equipo de trabajo en pro del sujeto de cuidado, familia y comunidad. La manera de ampliar y orientar el proceso de enseñanza-aprendizaje, permite al docente una mejor planeación, organización y comprensión de lo enseñado. La siguiente tabla resume qué debe alcanzar cada profesional para ser excelente en su rol. 
Tabla 2. Clasificación de competencias.

\begin{tabular}{|c|c|}
\hline COMPETENCIAS & CONCEPTO \\
\hline Básicas & Son aquellas que el estudiante adquirió en su formación básica y media. \\
\hline Genéricas & $\begin{array}{l}\text { Conocimientos generales que permiten al estudiante integrase a la socie- } \\
\text { dad; es decir, la forma de comportamiento y habilidades para el desempe- } \\
\text { ño profesional. }\end{array}$ \\
\hline Específicas & $\begin{array}{l}\text { Son aquellas propias de una disciplina o profesión, para el caso de las } \\
\text { Ciencias de la Salud, hay competencias únicas para el desarrollo y com- } \\
\text { prensión del ser humano. }\end{array}$ \\
\hline Laborales & $\begin{array}{l}\text { Permite integrar el conocimiento, habilidades y actitudes en el contexto } \\
\text { donde se desempeñe profesionalmente. }\end{array}$ \\
\hline
\end{tabular}

Fuente: (Pinilla, 2007) Con algunas modificaciones.

\section{La universidad concibe la evaluación como:}

Instrumento sistemático de recogida y análisis de información para emitir juicios y tomar decisiones, proporcione al profesor información para ajustar su intervención didáctica y que sirva no sólo para evaluar el progreso de los alumnos, sino también para orientar y guiar el proceso de enseñanza-aprendizaje. La evaluación debe ser formativa y la evaluación debe ser formadora en el proceso de enseñanzaaprendizaje, para lo cual se tiene en cuenta la autoevaluación o autocontrol cognitivo como evidencia o producto de fortaleza de aprendizaje cuya regulación está dada por el estudiante. La finalidad de la evaluación es formar para fortalecer el proceso de regulación y de autorregulación para llevar al estudiante a ser crítico y argumentativo frente a su desempeño profesional (Lineamientos 2012).

A la competencia la define como:

Conjunto de actitudes, valores, conocimientos y habilidades personales, interpersonales, profesionales y organizacionales que posibilitan el desempeño de los ciudadanos en el mundo de la vida y en el mundo del trabajo (Lineamientos 2012).

Las competencias a evaluar: El docente construye en el proyecto pedagógico de aula las competencias básicas que se espera que el estudiante alcance con el desarrollo del mismo, en tal sentido se definen de forma clara con el estudiante, los contenidos, habilidades, destrezas, principios y valores que serán objeto de evaluación, dado el carácter fundamental de los mismos para alcanzar las competencias planteadas.

La forma de evaluar: Son los medios que el docente utilice para obtener la información necesaria respecto a los avances en el proceso de enseñanza-aprendizaje. 
El momento de evaluar: El constructivismo plantea valorar la integridad del proceso formativo, momentos de la evaluación: inicial (diagnóstico de pre saberes, punto de partida para el inicio del proceso de aprendizaje), formativa (la cual se extiende a lo largo del proceso de formación y permite valorar el ritmo de cada estudiante y los avances alcanzados en el logro de las competencias definidas).

Por lo anterior, este estudio permitió una triangulación de la información para generar estrategias pedagógicas o planes de mejora.

\section{Materiales y métodos}

Se realizó un estudio descriptivo- transversal, el cual demanda un nivel de comprensión del objeto a estudiar para caracterizar e identificar categorías que permitan identificar representación verbal, numérica o gráfica; de cohorte transversal, a partir del cual se "analiza el fenómeno de interés en un momento particular" (Paramo, 2011).

Criterios de inclusión y exclusión: Población: profesores y estudiantes del programa de Enfermería.

Participantes y caracterización: Los participantes se buscaron por conveniencia, y fueron seleccionados a través del contacto personal y se vio el criterio de saturación de la información para definir el número de entrevistas. Participaron docentes con mínimo de tres años y al menos formación en educación o pedagogía a través de diplomados o programas formales de posgrado.

Los estudiantes participantes fueron los que cursaban asignaturas de cuidados de enfermería: al Adulto I, al Adulto II, a la Madre, al Niño y al Adolescente.

\section{Procedimiento}

Esta investigación contó con seis fases, en la primera fase se elaboraron entrevistas semiestructuradas a docentes del programa de enfermería, con unos criterios de selección; en la segunda fase: se llevó a cabo la validación de la guía de entrevista con ayuda de docentes de ciencias de la salud y expertos en educación; en la tercera fase se realizaron las entrevistas a docentes en un periodo alrededor de un mes. Posteriormente se procedió a realizar la transcripción, análisis, interpretación y codificación de cada una de las entrevistas. En la cuarta fase se elaboraron cuestionarios con preguntas cerradas para aplicarlos a estudiantes de enfermería con el fin de correlacionar las prácticas evaluativas del binomio (docente-estudiante); en la quinta fase se procedió al análisis de la información obtenida de los cuestionarios por pasos: organización, descripción e interpretación de datos. La sexta y última fase llevó a cabo la triangulación de la información encontrada a partir de los diferentes 
participantes: docentes, estudiantes y marco teórico (Cisterna, 2005).

Instrumentos para la recolección de datos: se realizó cuestionarios a estudiantes por correo electrónico y entrevistas a docentes previo consentimiento informado.

\section{Resultados}

La triangulación según Cisterna (2005) se entiende como la relación entre las diferentes interpretaciones elaboradas a partir de los hallazgos en el marco teórico, entrevistas y cuestionarios, buscando resolver la pregunta de investigación sobre la coherencia entre modelo pedagógico y las prácticas de evaluación. A continuación se describen las categorías emergentes del modelo pedagógico y evaluación.

Tabla 3. Triangulación de Modelo pedagógico.

\begin{tabular}{|c|c|c|c|}
\hline $\begin{array}{l}\text { CATEGORÍAS } \\
\text { DE MODELO } \\
\text { PEDAGÓGICO }\end{array}$ & MARCO TEÓRICO & $\begin{array}{l}\text { ENTREVISTAS A } \\
\text { DOCENTES }\end{array}$ & $\begin{array}{l}\text { CUESTIONARIOS A } \\
\text { ESTUDIANTES }\end{array}$ \\
\hline $\begin{array}{l}\text { Modelo de la } \\
\text { Universidad }\end{array}$ & $\begin{array}{l}\text { La universidad } \\
\text { estableció el modelo } \\
\text { Constructivista, } \\
\text { elementos } \\
\text { argumentativos, creativos } \\
\text { e investigativos. } \\
\text { El proceso de enseñanza } \\
\text { y aprendizaje requiere } \\
\text { que el estudiante realice } \\
\text { búsquedas bibliográficas, } \\
\text { analice la información } \\
\text { y para construir su } \\
\text { propio conocimiento, } \\
\text { siendo reflexivo, } \\
\text { argumentativo y crítico } \\
\text { para resolver problemas } \\
\text { reales; siempre } \\
\text { evaluando él, proceso } \\
\text { con retroalimentación } \\
\text { permanente del avance } \\
\text { del conocimiento del } \\
\text { estudiante (Flórez, 2005). }\end{array}$ & $\begin{array}{l}\text { Refieren que el } \\
\text { modelo es el } \\
\text { constructivista, } \\
\text { pero se videncia } \\
\text { que el utilizado en } \\
\text { el aula de clase es: } \\
\text { el conductista y } \\
\text { tradicional. }\end{array}$ & $\begin{array}{l}\text { No hay claridad en el } \\
\text { modelo pedagógico } \\
\text { implementado. No } \\
\text { conocen el modelo } \\
\text { pedagógico de la } \\
\text { institución. Hay } \\
\text { desconocimiento } \\
\text { de los lineamientos } \\
\text { institucionales. } \\
\text { El grupo de cuidado al } \\
\text { Adulto II, cuyo docente } \\
\text { explico de antemano el } \\
\text { perfil profesional de la } \\
\text { carrera y los referentes } \\
\text { pedagógicos responde } \\
\text { que el modelo es } \\
\text { constructivista. }\end{array}$ \\
\hline
\end{tabular}




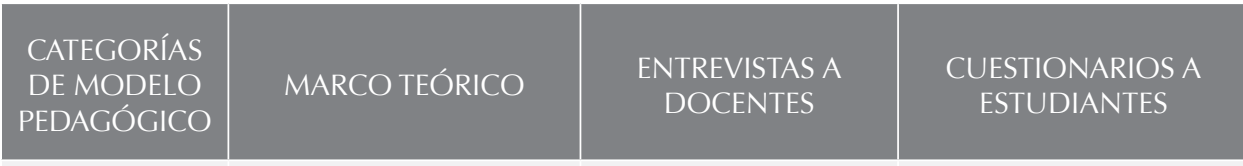

\begin{tabular}{|c|c|c|c|}
\hline Elementos & $\begin{array}{l}\text { Para Tamayo (2012) un } \\
\text { modelo pedagógico } \\
\text { está compuesto por } \\
\text { los fines, métodos, } \\
\text { relaciones maestro } \\
\text { alumno, objetivos, } \\
\text { conceptos de desarrollo } \\
\text { humano, valores, } \\
\text { sociedad, cultura, tipo de } \\
\text { currículo, concepciones } \\
\text { sobre el aprendizaje } \\
\text { y la evaluación, en } \\
\text { la Fundación está } \\
\text { establecido: Currículo, } \\
\text { estudiantes, docentes, } \\
\text { investigación y } \\
\text { proyección social. }\end{array}$ & $\begin{array}{l}\text { Están fragmentados } \\
\text { los elementos que } \\
\text { conforman el modelo } \\
\text { pedagógico y no } \\
\text { hay mucha claridad } \\
\text { en la finalidad, } \\
\text { aunque hablan de } \\
\text { la importancia en la } \\
\text { relación docente- } \\
\text { estudiante esta no se } \\
\text { pone en práctica en el } \\
\text { aula de clase. }\end{array}$ & $\begin{array}{l}\text { No conocen los } \\
\text { elementos del modelo } \\
\text { pedagógico. }\end{array}$ \\
\hline $\begin{array}{l}\text { Aspectos } \\
\text { enseñanza- } \\
\text { aprendizaje }\end{array}$ & $\begin{array}{l}\text { La investigación, el } \\
\text { saber científico, la } \\
\text { comunicación y la ética. } \\
\text { Orientando el proceso } \\
\text { a partir de los intereses } \\
\text { de los estudiantes, } \\
\text { teniendo en cuenta } \\
\text { el conocimiento } \\
\text { profesional del profesor. } \\
\text { La manera de lograr el } \\
\text { aprendizaje significativo } \\
\text { es mediante la evidencia } \\
\text { de la construcción } \\
\text { y transformación de } \\
\text { estructuras conceptuales, } \\
\text { actitudinales, prácticas } \\
\text { y axiológicas, y la } \\
\text { capacidad de interactuar } \\
\text { con el otro. }\end{array}$ & $\begin{array}{l}\text { Para los docentes } \\
\text { los aspectos son: la } \\
\text { didáctica, el currículo, } \\
\text { la investigación, } \\
\text { la evaluación; } \\
\text { sin embargo no } \\
\text { se incluye, la } \\
\text { comunicación y la } \\
\text { ética }\end{array}$ & $\begin{array}{l}\text { Para el estudiante es el } \\
\text { marco de referencia para } \\
\text { el proceso de enseñanza- } \\
\text { aprendizaje }\end{array}$ \\
\hline
\end{tabular}


Coherencia del modelo pedagógico y la evaluación del aprendizaje en una universidad de Bogotá

Tabla 4. Triangulación de Evaluación.

\begin{tabular}{|c|c|c|c|}
\hline $\begin{array}{c}\text { CATEGORÍAS } \\
\text { DE } \\
\text { EVALUACIÓN }\end{array}$ & MARCO TEÓRICO & ENTREVISTAS & CUESTIONARIOS \\
\hline $\begin{array}{l}\text { Modelo de } \\
\text { evaluación por } \\
\text { la Universidad }\end{array}$ & $\begin{array}{l}\text { Por competencias: La universidad } \\
\text { la define como un conjunto de } \\
\text { actitudes, valores, conocimientos } \\
\text { y habilidades personales, } \\
\text { interpersonales, profesionales y } \\
\text { organizacionales que posibilitan el } \\
\text { desempeño de los ciudadanos en } \\
\text { el mundo de la vida y en el mundo } \\
\text { del trabajo. Las competencias } \\
\text { hacen referencia a todo un } \\
\text { conjunto de conocimientos, } \\
\text { habilidades, destrezas, actitudes, } \\
\text { sentimientos y valores que se } \\
\text { requieren para desempeñar con } \\
\text { éxito o con un determinado } \\
\text { nivel de perfección, una tarea o } \\
\text { conjunto de tareas que responden } \\
\text { a las necesidades de una realidad } \\
\text { específica (Cerda, 2000). }\end{array}$ & $\begin{array}{l}\text { Conocen que deben } \\
\text { evaluar a partir del } \\
\text { enfoque formativo, } \\
\text { pero que por falta } \\
\text { de tiempo se ha } \\
\text { convertido sólo } \\
\text { en evaluación } \\
\text { sumativa, sin } \\
\text { permitirse verificar } \\
\text { el verdadero } \\
\text { significado a través } \\
\text { del aprendizaje. }\end{array}$ & $\begin{array}{l}\text { Los estudiantes } \\
\text { identifican que la } \\
\text { evaluación es para } \\
\text { conocer fortalezas } \\
\text { y debilidades en el } \\
\text { aprendizaje. }\end{array}$ \\
\hline Interpretación & $\begin{array}{l}\text { Basarse en otras opiniones para } \\
\text { interpretar datos cualitativos, } \\
\text { es decir, hacer uso de las } \\
\text { dimensiones de la coevaluación y } \\
\text { la heteroevaluación. }\end{array}$ & $\begin{array}{l}\text { Según los resultados } \\
\text { la interpretan de la } \\
\text { siguiente manera: } \\
\text { En la teoría: } \\
\text { heteroevaluación, } \\
\text { porque el tiempo } \\
\text { es más corto y hay } \\
\text { demasiada temática. } \\
\text { En la práctica: } \\
\text { coevaluación y } \\
\text { heteroevaluación, } \\
\text { se facilita por el } \\
\text { poco número de } \\
\text { estudiantes. }\end{array}$ & $\begin{array}{l}\text { Según los } \\
\text { resultados, se } \\
\text { evidencia más } \\
\text { heteroevaluación, } \\
\text { menos } \\
\text { coevaluacion. } \\
\text { Solo el docente } \\
\text { evalúa, sin permitir } \\
\text { la participación } \\
\text { activa del } \\
\text { estudiante, lo que } \\
\text { no ayuda a generar } \\
\text { redireccionamiento } \\
\text { de las falencias del } \\
\text { aprendizaje. }\end{array}$ \\
\hline
\end{tabular}




\begin{tabular}{|c|c|c|c|}
\hline $\begin{array}{c}\text { CATEGORÍAS } \\
\text { DE } \\
\text { EVALUACIÓN }\end{array}$ & MARCO TEÓRICO & ENTREVISTAS & CUESTIONARIOS \\
\hline Momentos & $\begin{array}{l}\text { Para la Universidad debe ser } \\
\text { continua: registra el desarrollo del } \\
\text { proceso de aprendizaje individual } \\
\text { a partir de las condiciones y los } \\
\text { avances propios de cada estudiante, } \\
\text { en el modelo constructivista del } \\
\text { proceso enseñanza aprendizaje. } \\
\text { La evaluación debe tener en } \\
\text { cuenta aspectos de enseñanza } \\
\text { del docente y el aprendizaje del } \\
\text { docente, para encaminar el proceso } \\
\text { a una reflexión tanto desde la } \\
\text { enseñanza hacia el aprendizaje, } \\
\text { como del aprendizaje hacia la } \\
\text { enseñanza; esto quiere decir que } \\
\text { requiere el proceso del estudiante } \\
\text { y del docente es conjunto. Para } \\
\text { cumplir con ello se debe propender } \\
\text { por generar autoevaluación, } \\
\text { coevaluación y heteroevaluación } \\
\text { (Díaz y Hernández, 2002). }\end{array}$ & $\begin{array}{l}\text { Los docentes se } \\
\text { refieren a que } \\
\text { evalúan en el } \\
\text { momento teórico } \\
\text { y práctico, pero } \\
\text { no manifiestan } \\
\text { claramente que ésta } \\
\text { es permanente o } \\
\text { continúa. }\end{array}$ & $\begin{array}{l}\text { Los estudiantes } \\
\text { refieren, en } \\
\text { general, que los } \\
\text { evalúan durante } \\
\text { todo el semestre. }\end{array}$ \\
\hline Propósito & $\begin{array}{l}\text { Para la Universidad: Formativa } \\
\text { y formadora: no tiene carácter } \\
\text { de sanción. Está orientada al } \\
\text { mejoramiento del proceso de } \\
\text { aprendizaje. } \\
\text { Llevar al estudiante hacia una } \\
\text { construcción nueva que le permita } \\
\text { un buen desarrollo profesional; } \\
\text { donde la relación docente/ } \\
\text { estudiante es más interactiva, la } \\
\text { cual le permite analizar, interpretar, } \\
\text { proponer y argumentar (Pinilla, } \\
\text { 2007). }\end{array}$ & $\begin{array}{l}\text { Los docentes } \\
\text { refieren que el } \\
\text { propósito es que } \\
\text { sea formativa, y } \\
\text { que su función es } \\
\text { diagnosticar los } \\
\text { conocimientos } \\
\text { previos del } \\
\text { estudiante, dentro } \\
\text { de otras funciones } \\
\text { mencionan la } \\
\text { pedagógica y social. } \\
\text { El propósito } \\
\text { general es para } \\
\text { que el estudiante } \\
\text { reconozca sus } \\
\text { debilidades y así } \\
\text { fortalecer y mejorar } \\
\text { su proceso de } \\
\text { aprendizaje. }\end{array}$ & $\begin{array}{l}\text { Para identificar } \\
\text { fortalezas y } \\
\text { debilidades en el } \\
\text { aprendizaje. } \\
\text { Para verificar } \\
\text { conocimientos por } \\
\text { parte del docente. }\end{array}$ \\
\hline
\end{tabular}




\begin{tabular}{|c|c|c|c|}
\hline $\begin{array}{c}\text { CATEGORÍAS } \\
\text { DE } \\
\text { EVALUACIÓN }\end{array}$ & MARCO TEÓRICO & ENTREVISTAS & CUESTIONARIOS \\
\hline Instrumentos & $\begin{array}{l}\text { Para la Universidad: Son los medios } \\
\text { que el docente utilice para obtener } \\
\text { la información necesaria respecto } \\
\text { a los avances en el proceso de } \\
\text { enseñanza-aprendizaje. } \\
\text { Díaz y Hernández (2002), } \\
\text { describe instrumentos formales, } \\
\text { semiformales e informales, lo } \\
\text { importante es que cualquiera que } \\
\text { se utilice marque la pauta en el } \\
\text { estudiante, Ilevándolo a reflexionar, } \\
\text { proponer o argumentar. }\end{array}$ & $\begin{array}{l}\text { Los docentes } \\
\text { utilizan técnicas } \\
\text { formales: como } \\
\text { parciales escritos, } \\
\text { estudios de } \\
\text { caso, mapas } \\
\text { conceptuales, pero } \\
\text { no le dan validez a } \\
\text { técnicas informales } \\
\text { como los trabajos } \\
\text { elaborados en casa, } \\
\text { porque no alcanza } \\
\text { el tiempo para } \\
\text { revisar. } \\
\text { No se evidencia } \\
\text { la técnica oral } \\
\text { para permitirle } \\
\text { al estudiante la } \\
\text { reflexión, critica, } \\
\text { argumentación y } \\
\text { proposición. Lo } \\
\text { anterior por temor } \\
\text { de sustentar en un } \\
\text { momento dado una } \\
\text { nota. }\end{array}$ & $\begin{array}{l}\text { Los estudiantes } \\
\text { manifiestan que } \\
\text { son evaluados } \\
\text { a través de las } \\
\text { pruebas escritas, } \\
\text { estudios de } \\
\text { caso y mapas } \\
\text { conceptuales; hay } \\
\text { otras herramientas } \\
\text { menos utilizadas } \\
\text { como: los ensayos, } \\
\text { aulas virtuales, } \\
\text { portafolios, } \\
\text { pruebas y trabajos } \\
\text { fuera del aula. }\end{array}$ \\
\hline $\begin{array}{l}\text { Aspectos a } \\
\text { evaluar }\end{array}$ & $\begin{array}{l}\text { Para la Universidad: El docente } \\
\text { construye en el proyecto } \\
\text { pedagógico de aula (PPA), las } \\
\text { competencias básicas que se } \\
\text { espera que el estudiante alcance } \\
\text { con el desarrollo del mismo, en } \\
\text { tal sentido, se definen, de forma } \\
\text { clara, con el estudiante, los } \\
\text { contenidos, habilidades, destrezas, } \\
\text { principios y valores que serán } \\
\text { objeto de evaluación, dado el } \\
\text { carácter fundamental de los mismos } \\
\text { para alcanzar las competencias } \\
\text { planteadas. }\end{array}$ & $\begin{array}{l}\text { Los docentes } \\
\text { evalúan a través de } \\
\text { las competencias: } \\
\text { el saber, el saber } \\
\text { hacer, lo actitudinal. } \\
\text { Aunque no se } \\
\text { evidencia en las } \\
\text { entrevistas si se } \\
\text { evalúa la forma } \\
\text { de comunicación } \\
\text { con el individuo, } \\
\text { familia, comunidad } \\
\text { y equipo de trabajo, } \\
\text { identificándose } \\
\text { mejor en el } \\
\text { momento practico, } \\
\text { es donde tiene todo } \\
\text { un contexto para } \\
\text { relacionarse. }\end{array}$ & $\begin{array}{l}\text { Los estudiantes } \\
\text { manifiestan que } \\
\text { el aspecto que } \\
\text { más prevalece } \\
\text { es la evaluación } \\
\text { de lo cognitivo, } \\
\text { es decir, el saber. } \\
\text { Pero también } \\
\text { se evidencia } \\
\text { evaluación a través } \\
\text { del saber hacer } \\
\text { en segundo lugar, } \\
\text { seguido por lo } \\
\text { actitudinal y la } \\
\text { comunicación con } \\
\text { el otro. }\end{array}$ \\
\hline
\end{tabular}


Se puede afirmar que existe una voluntad explícita desde el punto de vista institucional para diseñar y promover "un discurso oficial" sobre el modelo pedagógico desde el punto de vista conceptual y teórico. Este discurso se asume desde el constructivismo como una teoría cognitiva con profundas consecuencias para pensar la enseñanza.

Sin embargo, los docentes reivindican en sus respuestas una especie de mezcla de modelos de acuerdo con los contextos, grupos y asignaturas. Por otra parte, los estudiantes no tienen la "cultura acerca de los procesos de enseñanza", es decir: no se preguntan por los fines, los contenidos, los métodos, ni las formas de evaluación que define su currículo y se focalizan solamente en el ritual de transmisión de respuestas.

\section{Discusión y conclusiones}

La universidad plantea el enfoque constructivista, pero no se identifica en el proceso de enseñanza-aprendizaje, porque en el aula se lleva a cabo un modelo tradicional y conductista.

La evaluación debe ser integral, de competencias profesionales por procesos, pero se hace de modo sumativo. Se exploró el proceso de la evaluación al estudiante del programa de enfermería de la universidad, evidenciando que los estudiantes coinciden con las prácticas mencionadas por los docentes, pero se deja ver que no han tenido interés en conocer los lineamientos de la institución en la cual se están formando.

Al analizar el proceso enseñanza-aprendizaje desde el modelo pedagógico y las prácticas evaluativas, se evidenció que el $100 \%$ de docentes entrevistados tienen conocimientos en pedagogía, sin embargo, es necesario fortalecer continuamente las herramientas y estrategias pedagógicas para mejorar el quehacer docente.

\section{Recomendaciones}

Objetivo: Generar estrategias para mejorar el proceso evaluativo acorde al modelo pedagógico en el programa de enfermería. 
Coherencia del modelo pedagógico y la evaluación del aprendizaje en una universidad de Bogotá | Blanca Elpidia Tovar Riveros |

Tabla 5. Teorización: plan de mejoramiento

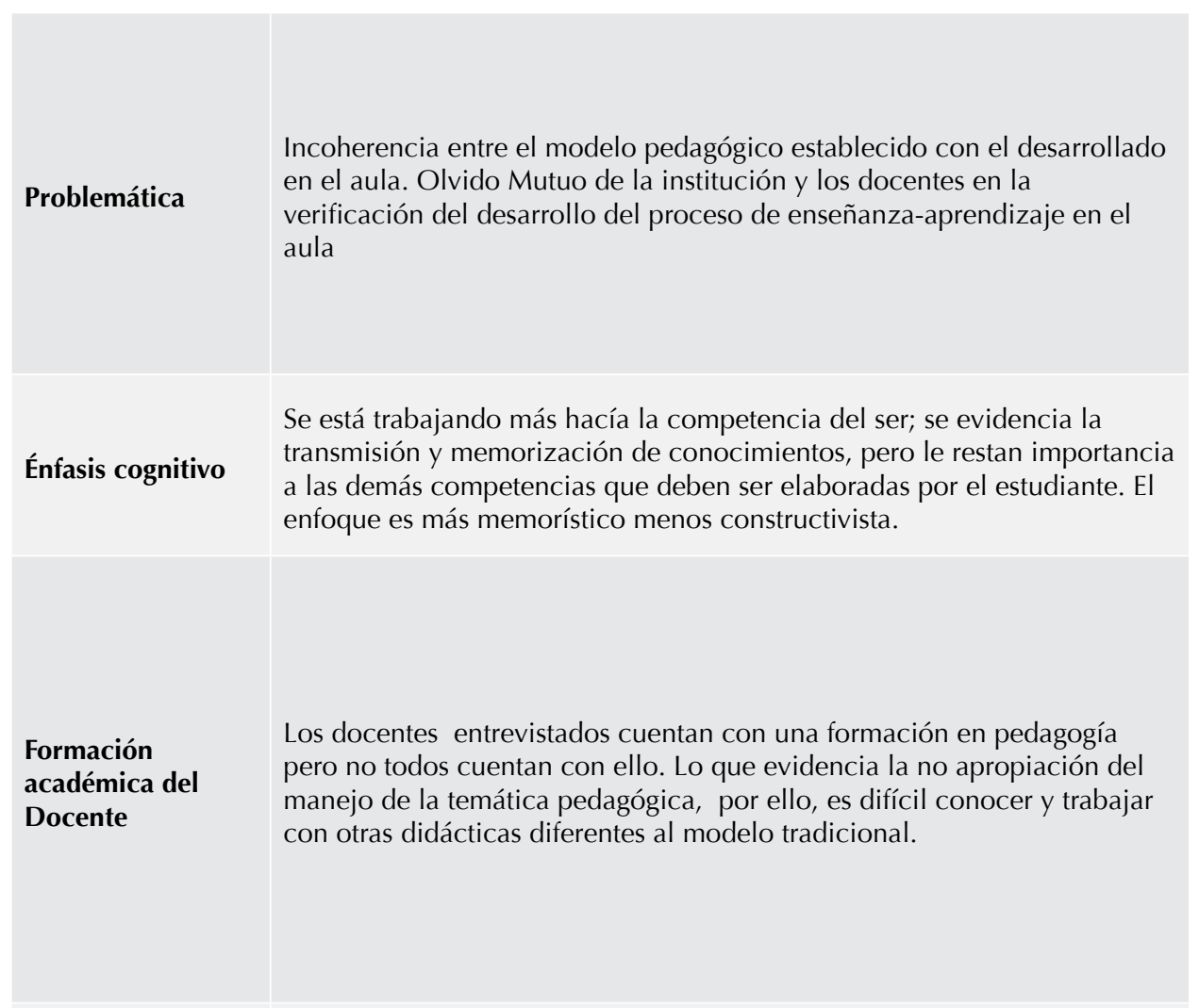

\section{Evaluación como calificación}

Por falta de tiempo, los docentes aunque conocen del propósito de la evaluación, no alcanzan a trabajarla como evaluación formativa y por procesos para que el estudiante alcance un aprendizaje significativo; si no que pasa a una función sumativa o terminal como opción de cumplimiento de la norma. 
Desmotivación por los docentes al no recibir un mejor pago y la falta de reconocimiento por parte de la institución para aquellos docentes que

Pago por mérito quieran capacitarse lo cual se podría evidenciar en el desarrollo acorde del proceso de enseñanza, que finalmente, se reflejará en un aprendizaje significativo en el estudiante mediante las competencias profesionales.

Posteriormente se generó propuesta para mejorar las prácticas evaluativas desde el modelo pedagógico.

Desarrollo:

1. Dar a conocer los lineamientos de la Universidad al grupo de docentes.

2. Capacitar al cuerpo docente en pedagogía para llevar a cabo en el aula una congruencia entre el modelo pedagógico, función y clase de evaluación para el mismo.

3. Implementar una materia en el primer semestre donde al estudiante se le confieran los lineamientos para su proceso enseñanza-aprendizaje. Con esto se busca concienciar al estudiante que va a realizar sus estudios en un modelo constructivista con un proceso de evaluación por competencias. 


\section{Referencias}

Cerda, H. (2000). La evaluación Como Experiencia Total. Logros-Objetivos-ProcesosCompetencias y Desempeño. Colombia: Cooperativa Editorial Magisterio.

Cisterna, F. (2005). Categorización y triangulación como procesos de validación del conocimiento en investigación cualitativa. Theoria 14(1), 61-71.

Díaz, F. y Hernández (2002). Estrategias Docentes Para Un Aprendizaje Significativo, Una Interpretación Constructivista. México: Mc Graw Hill.

Doblez, M.C., Céspedes, M., y García, J. (1995). Investigación en Educación-Procesos Interacciones y construcciones. Costa Rica: Primera Edición.

Flores, R. (2005). Pedagogía del Conocimiento. En: Modelos pedagógicos y enseñanza de las ciencias. Colombia. Editorial: McGraw Hill Interamericana. 2da. Edición.

Lineamientos de la Universidad. (2012). División de programación y desarrollo académico, la concepción institucional sobre la planeación de ambientes de aprendizaje en el modelo pedagógico constructivista por competencias, pdf: vac-da 012.

Gette, S.B. (2007). Fundamentación teórica de la evaluación de los aprendizajes a nivel superior. Barranquilla: Universidad Autónoma del Caribe.

Joint Committee on Standards for Educational Programs. Projects, and Materials (1981). Nueva York: Editorial McGraw- Hill.

Congreso de Colombia. (8 de febrero de 1994). Ley General de Educación. [Ley 115 de 1994]. Disponible en: http://www.banrepcultural.org/blaavirtual/educacion/leyedu/indice.htm

Mora, A. I. (2004). La Evaluación Educativa: Concepto, Períodos y Modelos. Actualidades Investigativas en Educación. 4, (2) p.4.Disponible en: http://www.mineducacion.gov. co/1621/articles-85906_archivo_pdf.pdf

Moreno, T. (2010). La evaluación de los alumnos en la universidad. Un estudio etnográfico. Pachuca: Universidad Autónoma del Estado de Hidalgo.

Paramo, P. (2008). La investigación en las ciencias sociales. Técnicas de recolección de Bogotá: Universidad Piloto de Colombia. 2da. edición

Parra, M. O. (2008). Generalidades de la Evaluación. En: reflexiones en Educación Universitaria II: Evaluación. Grupo de Apoyo Pedagógico y Formación Docente Facultad de Medicina. Bogotá: Universidad Nacional de Colombia. 2daEdición.

Perrenoud, P. (2004). Diez Nuevas Competencias Para Enseñar. Recuperado el 05 de junio de 2012 Disponible en: http://www.centrodemaestros.mx/enams/DiezCompetencias.pdf

Pinilla, A.E. (2007). Metodología de la evaluación académica. En: Reflexiones Sobre Educación Universitaria III. Grupo de Apoyo Pedagógico y Formación Docente Facultad de Medicina. Bogotá: Universidad Nacional de Colombia.

Sanmartí, N. (2007). 10 ideas clave-Evaluar para aprender. España: Graó. Edición: 1a 
Tema Libre

Tamayo, A. (2012). Acerca de los modelos pedagógico. El mapa no es el territorio, pero permite orientarnos en él. Seminarios investigación I. Maestría en educación Universidad Nacional de Colombia (Sin edición).

Recibido: 14 de julio 2015

Aceptado: 4 de septiembre 2015

Cómo citar: Tovar, B. (2016). Coherencia del modelo pedagógico y

la evaluación del aprendizaje en una universidad de Bogotá. Praxis

Pedagógica, 18, 53-70 